\title{
USING REPETITIVE CONTROL TO ELIMINATE PERIODIC DISTURBANCES IN DAMPER TEST RIGS
}

\author{
A. Plummer * J. Hätönen ${ }^{* *}$ D. H. Owens ${ }^{* *}$ \\ * Instron Ltd, Coronation Road, High Wycombe, \\ Bucks, HP12 3SY, UK \\ ${ }^{* *}$ Department of Automatic Control and Systems Engineering, \\ University of Sheffield, Mappin Street, Sheffield S1 3JD
}

\begin{abstract}
Repetitive Control is a relatively new technique for controlling systems that contain periodic reference signals or periodic disturbances. The fundamental idea behind the method is to use the internal model of the periodic signal to guarantee asymptotic reference tracking or disturbance rejection. In this paper two recent Repetitive Control algorithms are applied to a simulation model of a damper test rig. Damper test rigs are are widely used in the automotive industry to experimentally verify the mechanical durability properties of a given damper. These rigs, however, due to their mechanical setup, a tendency to generate internal periodic disturbances, which obviously have a negative effect on the reliability of the test results. The theoretical and simulation results in this paper show how Repetitive Control can be used to eliminate these periodic disturbances, resulting in a considerable increase in accuracy. Copyright $\odot 2005$ IFAC
\end{abstract}

Keywords: Repetitive Control, Optimal Control

\section{INTRODUCTION}

Many signals in engineering are periodic, or at least they can be accurately approximated by a periodic signal over a large time interval. This is true, for example, of most signals associated with engines, electrical motors and generators, converters, or machines performing a task over and over again. Hence it is an important control problem to try to track a periodic signal with the output of the plant or try to reject a periodic disturbance acting on a control system.

In order to solve this problem, a relatively new research area called Repetitive Control has emerged in the control community. The idea is to use information from previous periods to modify the control signal so that the overall system would 'learn' to track perfectly a given $T$-periodic reference signal or disturbance. The first paper that uses this ideology seems to be (Inouye et al., 1981), where the authors use repetitive control to obtain a desired proton acceleration pattern in a proton synchrotron magnetic power supply.

Since then repetitive control has found its way to several practical applications, including robotics (Kaneko and Horowitz, 1997), motors (Kobayashi et al., 1999), rolling processes (Garimella and Srinivasan, 1996) and rotating mechanisms (Fung et al., 2000). In this paper it is shown how Repetitive Control can be used to eliminate periodic disturbances in a damper test rig. To be more precise, two recent Repetitive Control algorithms are applied to a detailed simulation model of a damper test rig, which internally creates strong periodic disturbances due to the mechanical set-up of the system. The results show that using either of the two Repetitive Control Algorithms, the tracking performancance of the system is superior when compared to the results from a feedback controller.

The rest of the paper is outlined as follow: Section 2 introduces in detail the damper test rig framework 
and how it is used in the automotive industry. Section 3 gives a short review of the two Repetitive Control algorithms that are used in this paper. This followed by Section 4, which describes the simulation model used in this study. Section 5 presents the corresponding simulation results and analyses the performance of the Repetitive Control approach. Finally, Section 6 concludes the paper and gives directions for future research.

\section{DAMPER TEST RIG}

Structural testing is widely used to characterise the mechanical properties of structures and components, for example in the automotive and aerospace industries, see (Dodds and Plummer, 2001). Automotive test rigs may be designed to test components such as individual elastomeric bushes, or complete suspension systems, through to full vehicle test rigs (Dodds and Plummer, 2001). The purpose is either to characterise the dynamic properties of the test specimen, or to test for durability in a long term test.

Typically a structural test rig incorporates several hydraulic actuators which are controlled to impart loads or motion to the test specimen in a precise manner. However actuators are usually controlled using individual SISO PID controllers, with limited scope for reducing the effects of cross-axis interaction. The damper test rig considered in this paper highlights the problem and is described below.

Fig. 1 shows the rig. The damper is 'exercised' by a vertical actuator, while a side load is applied to the actuator body using a second actuator. Typically the vertical actuator is commanded with a sinewave of amplitude up to $100 \mathrm{~mm}$, and frequency up to around $20 \mathrm{~Hz}$. The side load command is usually a constant load of a few hundred Newtons. The problem is that the vertical motion couples into the side load causing a large load disturbance.

\section{REPETITIVE CONTROL}

Repetitive Control is a relatively new method to do high-quality control for systems that are associated with periodic signals. As a starting point in discretetime Repetitive Control (RC) it is assumed that a mathematical model

$$
\begin{aligned}
& \dot{x}(t)=A x(t)+B u(t) \\
& y(t)=C x(t)+D u(t)
\end{aligned}
$$

of the plant in question exists with $x(0)=x_{0}, t \in$ $[0, \infty)$. Furthermore, $A, B, C$ and $D$ are finitedimensional matrices of appropriate dimensions. From now on it is assumed that $D=0$, because in practice it very rare to find a system where the input function $u(t)$ has an immediate effect on the output variable $y(t)$. Furthermore, a reference signal $r(t)$ is given,

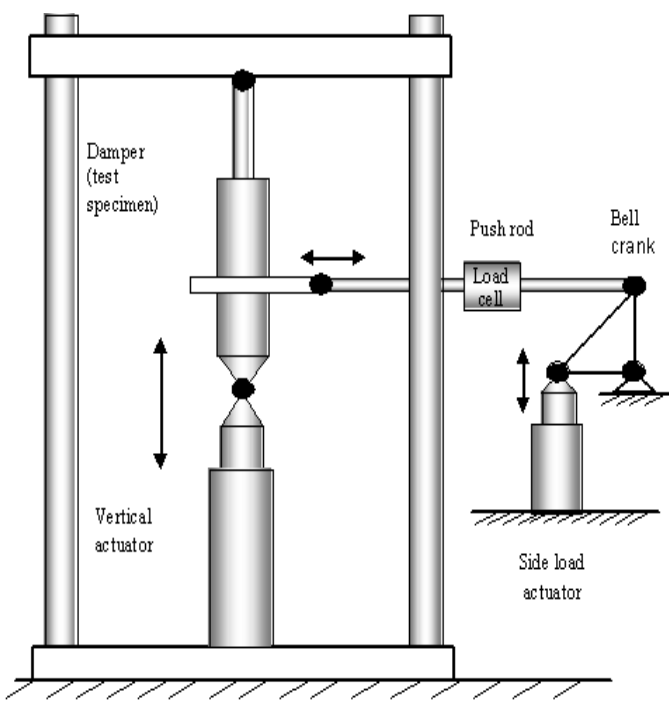

Fig. 1. Test rig

and it is known that $r(t)=r(t+T)$ for a given $T$ (in other words the actual shape of $r(t)$ is not necessarily known). The control design objective is to find a feedback controller that makes the system (1) to track the reference signal as accurately as possible (i.e. $\left.\lim _{t \rightarrow \infty} e(t)=0, e(t):=r(t)-y(t)\right)$, under the assumption that the reference signal $r(t)$ is $T$-periodic. As was shown by (Francis and Wonhan, 1975), a necessary condition for asymptotic convergence is that a controller

$$
[M u] t=[N e](t)
$$

where $M$ and $N$ are suitable operators, has to have an internal model or the reference signal inside the operator $M$. The notion 'internal model' means that if the operator $M$ is applied on $r(t)$ on $t \in(\infty, \infty)$, then $[M r](t)=0$. In the particular case when $r(t)$ is $T$-periodic, the internal model becomes $1-q^{-T}$, where $\left[q^{-T} v\right](t)=v(t-T)$ for $v: \mathbb{Z} \rightarrow \mathbb{R}$ (i.e. $q^{-1}$ is the standard left-shift operator), because

$$
\begin{aligned}
& \left(1-q^{-T}\right) r(k)=r(k)-q^{-T} r(k) \\
& =r(k)-r(k-T)=0
\end{aligned}
$$

on the infinite time-axis $t \in(\infty, \infty)$.

Thus in the discrete-time RC case the algorithm developement consists of selecting the operator $N(q)$ in the control law

$$
u(t)=N(q) \frac{1}{1-q^{-T}} e(t)
$$

so that the closed-loop system is stablised.

In this section two recent algorithms, namely optimality based and an adjoint type algorithm are shortly reviewed because they have been shown to solve robustly the design proplem proposed above. Furthermore, they are applied to the damper test rig in the following sections. For a more detailed description of the algorithms see (Hätönen et al., 2003), (Freeman et al., 2004) and (Hätönen et al., 2004). 


\subsection{Optimal algorithm}

In the optimal algorithm the idea is to translate the original system model

$$
A(q) y(k)=B(q) u(k)
$$

to a regulation problem using the internal model $D(q)=1-q^{-T}$ of the reference signal $r(t)$. To see how this can be done, multiply the process model from the left with $D(q)$. This results in

$$
\begin{aligned}
& D(q) A(q) y(k)=D(q) B(q) u(k) \\
& =A(q) D(q) y(k)=A(q)(y(k)-y(k-T)) \\
& =A(q)(-r(k)-r(k-T)+y(k)-y(k-T)) \\
& =-A(q)(e(k)-e(k-T))=-D(q) A(q) e(k) \\
& =B(q) D(q) u(k)
\end{aligned}
$$

Using the notation $\tilde{u}(k):=u(k)-u(k-T)$ the process model becomes

$$
-D(q) A(q) e(k)=B(q) \tilde{u}(k)
$$

which is now in the form of a standard regulator problem, i.e. find $\tilde{u}(k)$ so that $\lim _{k \rightarrow \infty} e(k)=0$. One way of achieving this is to form a state-space model for the modified plant model (7), resulting in

$\left\{\begin{array}{l}x_{m}(k+1)=A_{m} x_{m}(k)+B_{m} \tilde{u}(k), x_{m}(0)=x_{m, o} \\ e(k)=C_{m} x(k)\end{array}\right.$

and use LQR control to stablilise the 'extended' statespace model (8). To be more precise, in LQR the following optimisation problem is considered

$$
\left\{\begin{array}{l}
\min _{\tilde{u} \in l_{2}} J(\tilde{u}) \\
J(\tilde{u}):=\sum_{i=1}^{\infty}\left(e(i)^{T} Q e(i)+\tilde{u}(i)^{T} R \tilde{u}(i)\right)
\end{array}\right.
$$

where $Q$ and $R$ are positive-definite weighting matrices, and the corresponding solution is a state-feedback law

$$
\tilde{u}(k)=-K_{m} x_{m}(k)
$$

where $K_{m}$ is the solution of a Riccati-equation, see (Lewis and Syrmos, 1995). However, the state $x_{m}(\cdot)$ cannot obviously be measured directly. Unfortunately, in practise it is impossible to measure the state $x_{m}(\cdot)$ directly. However, it is still possible to construct an observer for the state $x_{m}(\cdot)$, i.e. the states are estimated with the following equation

$\hat{x}_{m}(t+1)=\Phi_{m} \hat{x}_{m}(t)+\Gamma_{m} \tilde{u}(t)+L\left(e(t)-C_{m} x_{m}(t)\right)$

where $L$ is the observer gain and the control law becomes

$$
u(t)=u(t-T)-K \hat{x}_{m}(t)
$$

Note that it is easy to take noise into account in the proposed algorithm: suppose that (8) would also have noise terms $w(t)$ and $v(t)$ in the following way,

$$
\begin{aligned}
& x_{m}(t+1)=\Phi_{m} x_{m}(t)+\Gamma_{m} \tilde{u}(t)+H w(t) \\
& e(t)=C_{m} x_{m}(t)+v(n)
\end{aligned}
$$

where $w(t)$ and $v(t)$ are zero mean Gaussian noise and $\quad x_{m}(0)=x_{m, o}$. Conceptually $w(t)$ describes

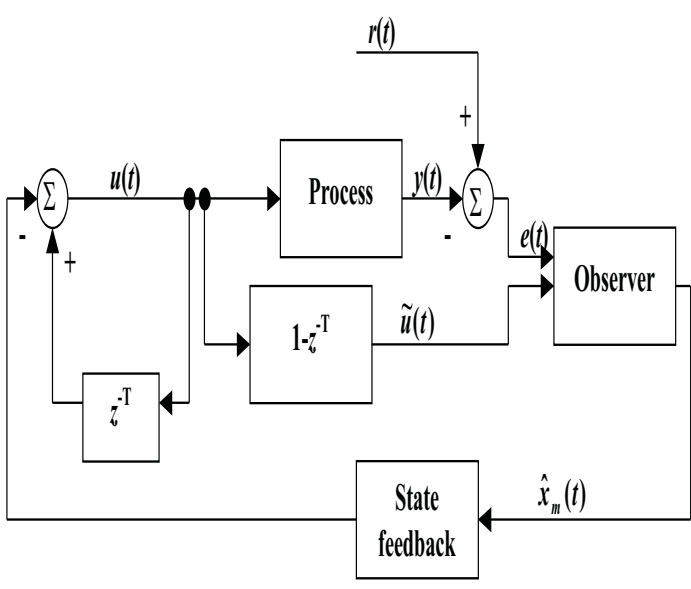

Fig. 2. Flow diagram for the optimality based RC algorithm

uncertainty in the state-space model, whereas $v(t)$ describes uncertainty in the measurement process. If the covariance matrix $Q_{n}$ of $v(t)$ and the covariance matrix $R_{n}$ of $w(t)$ are known, it is possible to find an optimal observer gain $L$ that minimises the variance of the estimation error. It is also a standard result in optimal control (see (Lewis and Syrmos, 1995)) that by combining the optimal feedback controller and optimal observer the resulting closed loop system is stable, and hence the expected value of $e(t)$ will go exponentially to zero as $t \rightarrow \infty$.

Note that when the control law is implemented on the original plant (5), the control law (10) reads $u(k)=$ $u(k-T)+K_{m} \hat{x}_{m}(k)$, where $\hat{x}(\cdot)$ is now the estimated state of (8). Note also that a similar developement can be easily carried out for the case when the dynamical system has a $T$-periodic load disturbance. Fig. 2 shows the flow diagram for the optimality based $\mathrm{RC}$ algorithm.

\subsection{Adjoint based-based Repetitive Control}

Consider again the plant model (5) which is now written in the form

$$
\begin{aligned}
& y(k)=\frac{B(q)}{A(q)} u(k):=G(q) u(k) \\
& =h_{1} q^{-1} u(k)+h_{2} q^{-2} u(k)+\cdots
\end{aligned}
$$

where $h_{i}$ are the Markov-parameters of the system. In the the adjoint Repetitive Control based-algorithm the idea is to use the control law

$$
u(k)=q^{-T} u(k)+\beta q^{-T} G\left(q^{-1}\right) e(k)
$$

where

$$
G\left(q^{-1}\right)=h_{1} q+h_{2} q^{2}+\ldots
$$

This results in the error evolution equation

$$
e(k)=q^{-T}\left(1-\beta G(q) G\left(q^{-1}\right)\right) e(k)
$$

A reasonably staightforward application of the the Nyquist stability condition results in the following suf- 
ficient condition for asymptotic convergence to zero tracking error

$$
\left.\sup _{\omega \in[0,2 \pi]}|1-\beta| G\left(e^{j \omega}\right)\right|^{2} \mid<1
$$

which can be always met, if $\beta$ is taken to be sufficiently small. The problem, however, is that the algorithm (15) is typically non-causal, i.e. $u(t)$ is a function of future values of $e(t)$. An intuitive way to avoid this problem is truncate the impulse response of the plant model after $T$ steps, i.e. it is assumed that $h_{i}=0$ for $i>T$, resulting in a nominal finite impulse response model $G_{o}(q)=h_{1} q^{-1}+h_{2} q^{-2}+\cdots+$ $h_{T} q^{-T}$. In this case the algorithm (15) becomes

$$
u(k)=q^{-T} u(k)+\beta q^{-T} G_{o}\left(q^{-1}\right) e(k)
$$

and the corresponding error evolution equation is given by

$$
\begin{aligned}
& e(k)=q^{-T}\left(1-\beta G(q) G_{o}\left(q^{-1}\right)\right) e(k) \\
& =q^{-T}\left(1-\beta U(q) G_{o}(q) G_{o}\left(q^{-1}\right)\right) e(k)
\end{aligned}
$$

where $U(q)$ is the multiplicative uncertainty caused by truncation. Consequently it is important to analyse how $U(q)$ affects the convergence properties of the dynamical system (20). In (Hätönen et al., 2004) it has in fact been shown that if the phase of $U(q)$ lies in between $\pm 90^{\circ}$, then the adjoint based algorithm will still converge asymptotically to zero tracking error.

\section{SIMULATION MODEL OF THE DAMPER TEST RIG}

A dynamical simulation model of the damper test rig has been developed by Instron Ltd, which incorporates:

1) the hydraulic servovalve and actuator for the sideload system

2) the mechanical coupling between vertical motion and the sideload system

3) the PID controller for the side load actuator

The vertical actuation system is just represented by the sinusoidal motion it produces; a detailed model is not required to test the repetitive control algorithms. For the sideload system, the valve is modelled using conventional orifice flow equations with second order spool dynamics, and the actuator includes oil compressibility effects. Other practical phenomena such as a valve slew rate limit and cross-piston leakage are also included. The model is implemented in Simulink. For testing the repetitive control algorithms, a vertical excitation of $100 \mathrm{~mm}$ amplitude at $10 \mathrm{~Hz}$ is used.

\section{SIMULATION RESULTS}

Fig. 4 shows the performance of the test rig when it is controlled with a standard PID-controller. The PIDcontroller settings have been chosen to reflect settings that the rig operators would normally find via manual

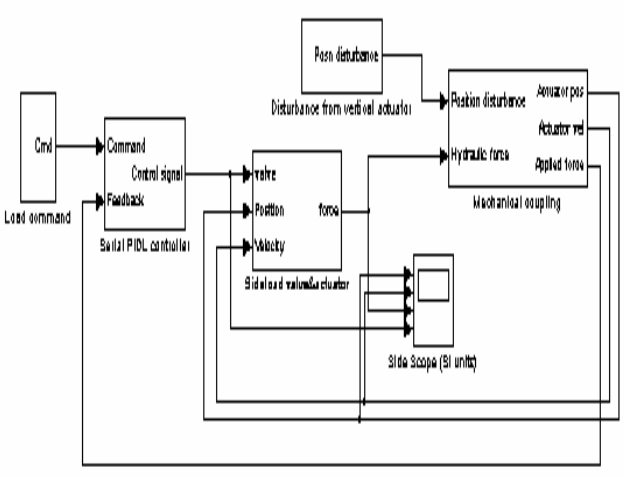

Fig. 3. Simulink model of the test rig

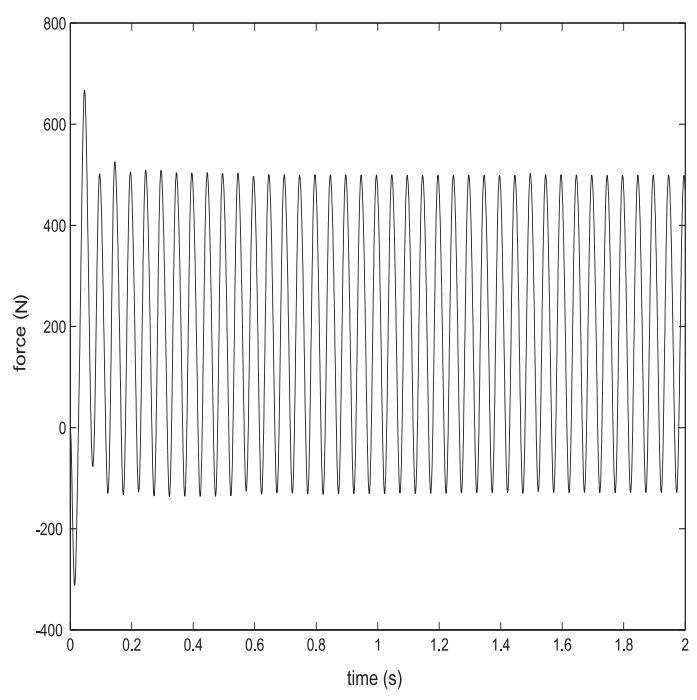

Fig. 4. Tracking behaviour with a PID controller

tuning. The set-point for the controller is $200 \mathrm{~N}$. As can be seen from this figure, the disturbances caused by the side load result in considerable oscillations around the set-point.

\subsection{Optimality based algorithm}

In this simulation the state-space model of the plant was identified using a standard subspace identification routine. The data for the subspace identification was obtained by exciting the system with white noise. Note that in this simulation the algorithm modifies the reference signal to the PID controller, i.e. the plant seen by the Repetitive Control algorithm is the closedloop plant. Fig. 5 shows the simulation results with the optimality based algorithm. The various parameters in the LQR contoller and Kalman filter were tuned manually to maximise convergence speed. The algorithm converges inside 0.4 seconds, demonstrating sufficient convergence speed for this particular application. 


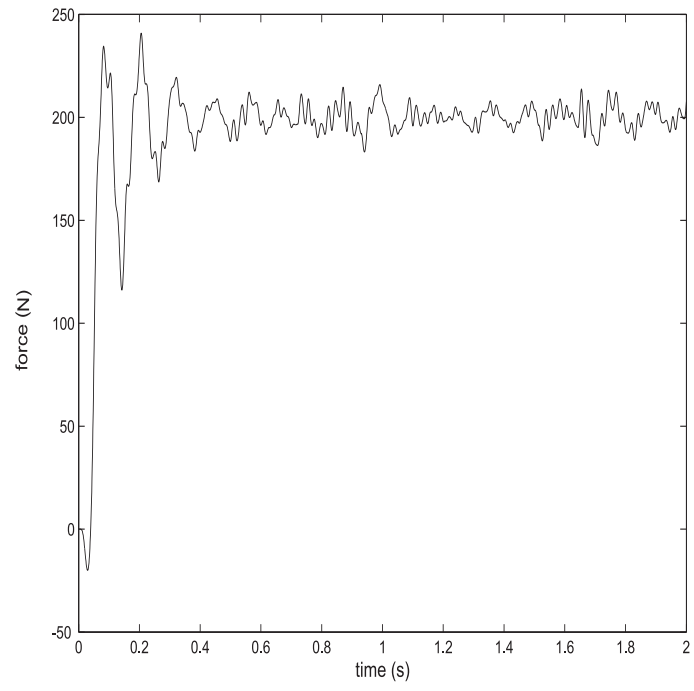

Fig. 5. Convergence behaviour with the optimal algorithm

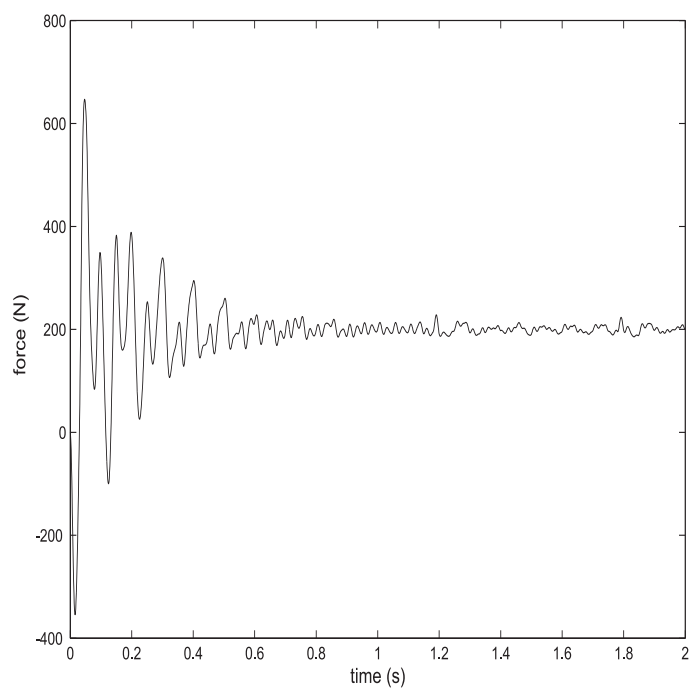

Fig. 6. Convergence behaviour with the adjoint algorithm

\subsection{Adjoint based algorithm}

The plant model $G_{o}(q)$ for the adjoint algorithm was obtained from an impulse-response experiment on the closed-loop plant. Fig. 6 shows how the adjoint algorithm performs with a constant set-point $200 \mathrm{~N}$ when the learning gain $\beta=1.2$. The learning gain was selected so that the learning rate would be maximised. The algorithm converges sufficiently fast for this particular application, but it is slightly slower than the optimality based algorithm.

\section{CONCLUSIONS AND FUTURE WORK}

From the simulation results, it is clear that both the optimality-based algorithm and the adjoint-based algorithm significantly reduce the disturbance on the side load caused by the vertical excitation. Without repetitive control, the error is about $\pm 300 N$; with either repetitive control algorithm this is reduced to about $\pm 10 N$. The optimality based algorithm converges more quickly at the expense of more involved system identification.

It is clear that repetitive control should be able to dramatically reduce errors where a periodic excitation interacts with other axes in a structural test rig. This now needs to be verified in practice. Consequently future work consists of implementing the algorithms in real-time using a suitable microprosessor environment and applying them to a structural test rig.

\section{ACKNOWLEDGEMENTS}

J. Hätönen has been supported by the EPSRC contract No GR/R74956/01.

\section{REFERENCES}

Dodds, C.J. and A.R. Plummer (2001). Laboratory road simulation for full vehicle testing - A Review. In: SIAT 2001-SAE Conferencel. India.

Francis, B.A. and W.M. Wonhan (1975). The internal model principle for linear multivariable regulators. Appl. Math. Opt. pp. 107-194.

Freeman, C., J. Hätönen, D.H. Owens, P. Lewin and E. Rogers (2004). A novel repetitive control algorithm combining ilc and dead-beat control. In: Proceedings of the International Conference on Informatics in Control, Automation and Robotics. Sebutal, Portugal.

Fung, R.F., J.S Huang, C.G. Chien and Y.C. Wang (2000). Design and application of continuous time controller for rotation mechanisms. International Journal of Mechanical Sciences 42, 18051819.

Garimella, S.S. and K. Srinivasan (1996). Application of Repetitive Control to eccentricity compensation in rolling. Journal of Dynamic Systems Measurement and Control-Transactions of the ASME 118, 657-664.

Hätönen, J., C. Freeman, D.H. Owens, P. Lewin and E. Rogers (2004). Robustness analysis of a gradient-based repetitive control algorithm for discrete-time systems. In: Proceedings of the 43rd IEEE Conference on Decision and Control. Atlantis, Paradise Island, Bahamas.

Hätönen, J., D.H. Owens and R. Ylinen (2003). A new optimality based repetitive control algorithm for discrete-time systems. In: Proceedings of the European Control Conference (ECCO3). Cambridge, UK.

Inouye, T., M. Nakano, T. Kubo, S. Matsumoto and H. Baba (1981). High accuracy control of a proton synchrotron magnet power supply. In: Proceedings of the 8th IFAC World Congress. Kyoto, Japan. 
Kaneko, K. and R. Horowitz (1997). Repetitive and adaptive control of robot manipulators with velocity estimation. IEEE Trans. on robotics and automation 13, 204-217.

Kobayashi, Y., T. Kimuara and S. Yanabe (1999). Robust speed control of ultrasonic motor based on $H_{\infty}$ control with repetitive compensator. JSME Int. Journal Series C 42, 884-890.

Lewis, F. L. and V. L. Syrmos (1995). Optimal Control. John Wiley \& Sons, Inc. 